\title{
Sex-Steroid Hormone Manipulation Reduces Brain Response to Reward
}

\author{
Julian Macoveanu $^{*, 1,2,3}$, Susanne Henningsson ${ }^{1,2}$, Anja Pinborg ${ }^{4,5,6}$, Peter Jensen $^{2,4}$, Gitte M Knudsen $^{2,4}$, \\ Vibe G Frokjaer ${ }^{2,3,4,8}$ and Hartwig R Siebner ${ }^{1,7,8}$
}

'Danish Research Centre for Magnetic Resonance, Copenhagen University Hospital, Hvidovre, Denmark; ${ }^{2}$ Center for Integrated Molecular Brain Imaging, Copenhagen University Hospital Rigshospitalet, Copenhagen, Denmark; ${ }^{3}$ Psychiatric Center Copenhagen, Copenhagen University Hospital Rigshospitalet, Copenhagen, Denmark; ${ }^{4}$ Neurobiology Research Unit, Copenhagen University Hospital Rigshospitalet, Copenhagen, Denmark;

${ }^{5}$ Fertility Clinic, Copenhagen University Hospital Rigshospitalet, Copenhagen, Denmark; ${ }^{6}$ Gynecology and Obstetrics, Copenhagen University Hospital, Hvidovre, Denmark; ' Department of Neurology, Copenhagen University Hospital, Copenhagen, Denmark

\begin{abstract}
Mood disorders are twice as frequent in women than in men. Risk mechanisms for major depression include adverse responses to acute changes in sex-steroid hormone levels, eg, postpartum in women. Such adverse responses may involve an altered processing of rewards. Here, we examine how women's vulnerability for mood disorders is linked to sex-steroid dynamics by investigating the effects of a pharmacologically induced fluctuation in ovarian sex steroids on the brain response to monetary rewards. In a double-blinded placebo controlled study, healthy women were randomized to receive either placebo or the gonadotropin-releasing hormone agonist (GnRHa) goserelin, which causes a net decrease in sex-steroid levels. Fifty-eight women performed a gambling task while undergoing functional MRI at baseline, during the mid-follicular phase, and again following the intervention. The gambling task enabled us to map regional brain activity related to the magnitude of risk during choice and to monetary reward. The GnRHa intervention caused a net reduction in ovarian sex steroids (estradiol and testosterone) and increased depression symptoms. Compared with placebo, GnRHa reduced amygdala's reactivity to high monetary rewards. There was a positive association between the individual changes in testosterone and changes in bilateral insula response to monetary rewards. Our data provide evidence for the involvement of sex-steroid hormones in reward processing. A blunted amygdala response to rewarding stimuli following a rapid decline in sex-steroid hormones may reflect a reduced engagement in positive experiences. Abnormal reward processing may constitute a neurobiological mechanism by which sex-steroid fluctuations provoke mood disorders in susceptible women.
\end{abstract}

Neuropsychopharmacology (2016) 4I, I057-1065; doi: I0.I038/npp.2015.236; published online 2 September 2015

\section{INTRODUCTION}

Cumulating evidence from both human and animal studies indicates that sex-steroid hormones such as estradiol, progesterone, and testosterone are involved in the neurofunctional modulation of the reward system and influence reward behavior (Dreher et al, 2007; Brummelte and Galea, 2010; Bayer et al, 2013; Peper et al, 2013). Fluctuations in ovarian steroids during the natural menstrual cycle have been reported to modulate reward-related activity in several brain regions such as the orbitofrontal cortex and amygdala (Dreher et al, 2007; Bayer et al, 2013).

\footnotetext{
*Correspondence: Dr J Macoveanu, Danish Research Centre for Magnetic Resonance, Centre for Functional and Diagnostic Imaging and Research, Copenhagen University Hospital Hvidovre, Kettegaard Alle 30, Hvidovre DK-2650, Denmark, Tel: +0045 3195 3196,

E-mail: julianm@drcmr.dk

${ }^{8}$ These authors contributed equally to this work.

Received II June 2015; revised 16 July 2015; accepted 27 July 20 I5; accepted article preview online 6 August 2015
}

Mood disorders manifest twice as frequently in women than in men, but it is unknown whether the increased frequency is related with sex-steroid dynamics. Larger fluctuations in endogenous ovarian steroids experienced by women, eg, postpartum and during menopausal transition, are indeed sometimes associated with severe mood state changes and an increased vulnerability for mood disorders (Freeman et al, 2006; Munk-Olsen et al, 2006). Such changes often involve depressed mood and anhedonia, ie, a reduced interest or pleasure in normally rewarding activities (Freeman et al, 2004). This is a core feature in the pathophysiology and symptomatology of several psychiatric disorders including major depression (Clark et al, 1994; Vrieze et al, 2013). Consistent with human studies, rodent models of postpartum depression show a reduced preference for drinking sucrose following ovarian steroid withdrawal, which is alleviated by treatment with estradiol (Brummelte and Galea, 2010). The anhedonic feature of depression has been associated with reduced ability to learn from rewarding events (Vrieze et al, 2013) and to a blunted amygdala response to positive emotional stimuli (Keedwell et al, 2005; 
Stuhrmann et al, 2013). Although these studies corroborate the critical role sex-hormone milieu has in stabilizing mood, the direct impact of large changes in sex-steroid dynamics on how rewards are processed by the human brain remains unknown.

Here, we provide an intervention framework for directly studying whether sex-steroid manipulations modulate human reward circuitry in order to advance our knowledge on sex-steroid-related risk mechanisms for neuropsychiatric disorders. Specifically, we investigated how a pharmacological manipulation of endogenous ovarian sex steroids affects brain response to risk-taking behavior and monetary rewards in healthy premenopausal women. The sex-steroid hormone levels were manipulated by administration of a gonadotropin-releasing hormone agonist (GnRHa), which first leads to an increase followed by a rapid decline in endogenous ovarian sex steroids.

Using a double-blinded parallel-group design, healthy female participants receive either a GnRHa implant or a placebo. We measured depression scores, serum estradiol, progesterone, and testosterone levels at baseline during midfollicular phase and at follow-up after the intervention. On both occasions, the participants underwent functional MRI while performing a card gambling task. The task prompted them to make risky gambling choices followed by either a monetary reward proportional to the risk or an invariable loss. We assessed the effect on the GnRHa intervention on the neural response to risky choices, and positive and negative outcomes. We hypothesized that a significant decline in ovarian hormone production would alter the neural response to monetary rewards in amygdala. Amygdala expresses high densities of sex-steroid receptors (Barth et al, 2015) showing sensitivity to normal variations in ovarian sex steroids (Toffoletto et al, 2014), and is critically involved in reward learning as well as processing positive and negative emotional reactions (Murray, 2007). We further explored whether the baseline to follow-up changes in neural response correlate with changes in the net levels of estradiol, testosterone, and depression scores following GnRHa intervention.

\section{MATERIALS AND METHODS}

\section{Study Design, Participants, and Intervention}

Sixty healthy young women (mean age $24.3 \pm 4.9$ years) with regular menstrual cycles between 23 and 35 days participated in the study and received modest financial compensation. The participants were recruited by advertisement for participation in a large neuroimaging study, including both molecular and functional MRI investigations. Data on molecular imaging of the serotonin transporter are presented in Frokjaer et al (2015). The participants were screened by trained clinicians regarding their medical history. This assessment included past and current psychiatric symptoms using the Schedules for Clinical Assessment in Neuropsychiatry (SCAN 2.1), premenstrual dysphoric disorder (DSM-IV), and alcohol, tobacco, and drug use. The participants underwent a neurological and gynecological examination. Following positive assessment, the participants reported the first menstrual day of their next cycle and were examined with ultrasound at cycle day 21 or 22 to confirm a postovulatory phase before initiating the pharmacological GnRHa intervention. All participants had normal blood tests including follicle-stimulating hormone, thyroid-stimulating hormone, and androgen status at baseline.

In a double-blinded, parallel study design, the participants were randomized to receive either $3.6 \mathrm{mg}$ GnRHa (goserelin) implant or saline injection during the midluteal phase (at a single occasion at cycle day $22.6 \pm 2.5$ ) by a gynecologist not involved in any subsequent interaction with the participants, data collection, or analysis to secure effective blinding. At the administered GnRHa dose, the HPG-axis is first stimulated and subsequently downregulated for an average of 28 days. The participants were investigated twice. Baseline assessments were performed in the midfollicular phase when ovarian hormone levels are most stable and the time elapsed since the postovulatory estradiol drop was maximal (cycle day $6.6 \pm 2.2)$, and at follow-up $(16.2 \pm 2.6$ days after intervention) using the same protocol. The follow-up investigation was positioned post bleeding at a time point late enough to allow the GnRHa group to have entered their early ovarian suppression phase. The protocol included a functional MRI scan (see below), assessment of depression symptoms according to the Hamilton 17-item depression rating scale (HAMD) (Hamilton, 1980), and measurements of serum estradiol, progesterone, and testosterone. The participants and the investigators involved in data acquisition and analysis were blinded to the intervention group until completion of the functional MRI analysis. Blinding was efficient as both the interviewer and the participants' guesses on group assignment did not differ significantly from chance during the period when imaging and psychometric data were acquired. One participant was excluded from the study owing to anovulation and one owing to excessive movement during the MRI investigation. In addition, three participants missed data from the follow-up fMRI investigation: one became pregnant, one missed the scheduled investigation day, and one dataset was lost owing to technical error. Consequently, 58 datasets were available for the baseline analysis and 55 for the longitudinal analysis (GnRHa group $n=26$, placebo group $n=29$ ). The study was registered and approved by the local ethics committee in Copenhagen under the protocol number H-2-2010-108. Written informed consent was obtained from all participants.

\section{Hormone Measurements}

The serum collected was delivered immediately to the hospital laboratory and kept at $5{ }^{\circ} \mathrm{C}$ until assayed within $24 \mathrm{~h}$. Estradiol, progesterone, and testosterone concentrations were determined by the Electrochemiluminescence Immunoassay method on Modular Analytics Serum Work Area equipment (Roche, Mannheim, Germany). Detection ranges were as follows: estradiol: $(0.04 ; 78.9 \mathrm{nmol} / \mathrm{l})$, progesterone: $(0.095 ; 191 \mathrm{nmol} / \mathrm{l})$, and testosterone $(0.42$; $52 \mathrm{nmol} / \mathrm{l})$. Hormone concentrations measured below the detection limit were set to the value of the lower limit.

\section{Functional MRI Data Analysis}

The participants performed a gambling task (Figure 1a and b) while undergoing fMRI at 3T. For detailed task descrip- 


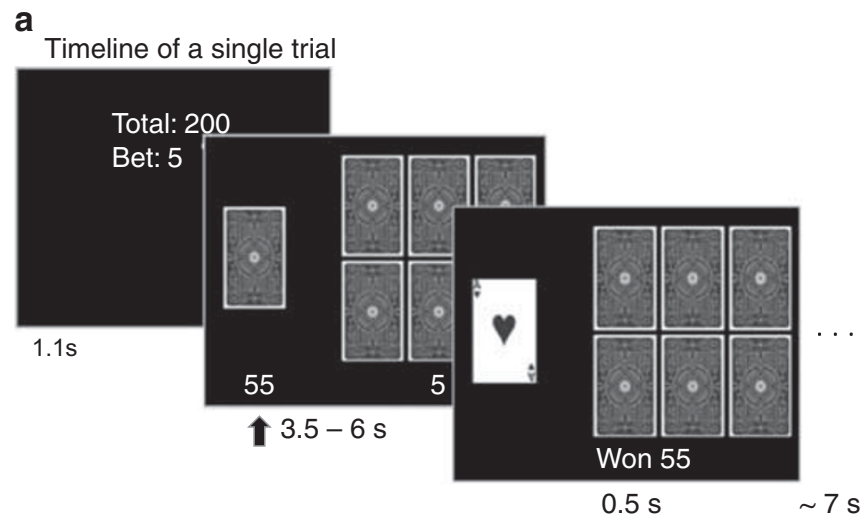

C Risk taking behavior
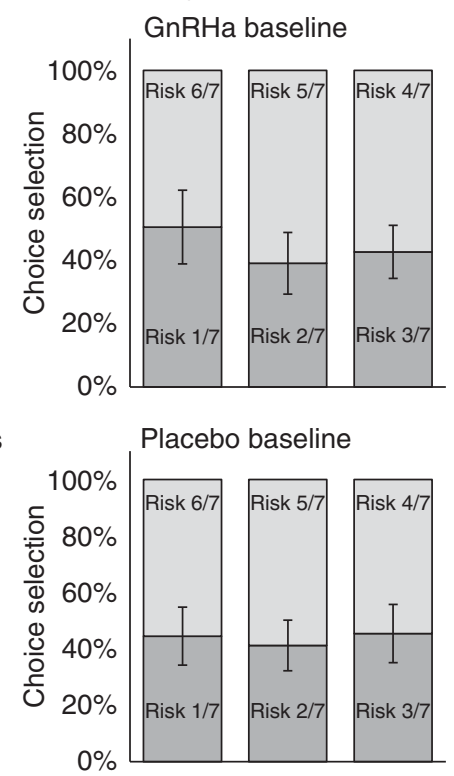

GnRHa follow-up

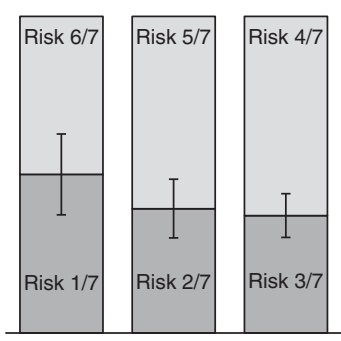

Placebo follow-up

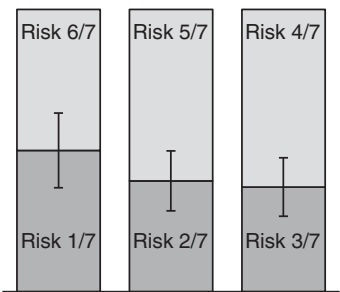

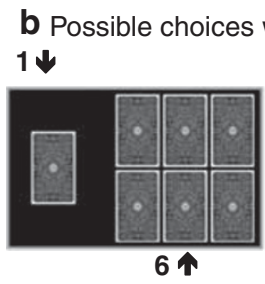

$33 / 44 / 55 \quad 3 / 4 / 5$

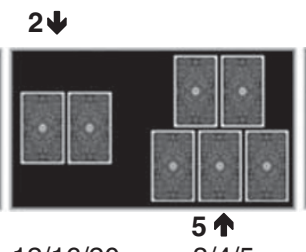

$12 / 16 / 20$

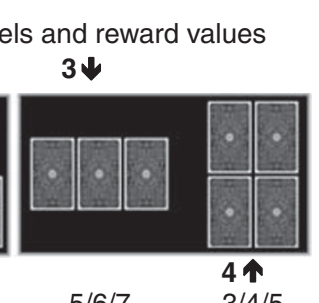

Figure I The card-gambling task. (a) All trials had three phases: Information, Choice and Outcome. During the Information phase, participants were informed about the sum of money they had accumulated and the bet size (3, 4, or 5 DKK), which could be lost. In the Choice phase, two sets of cards were presented together with the associated monetary reward below. Participants chose the set of cards where they believed the 'Ace of hearts' would be hidden. In the Outcome phase, the 'Ace of hearts' was revealed, providing the participants a feedback whether they chose the right set and won the associated reward or lost the bet. Figure adapted with permission from Macoveanu et al (20 I3a). (b) The six possible choices with associated winning amounts in DKK. Figure adapted with permission from Macoveanu et al (20/3a). (c) Risk choice behavior during the gambling task. The panel shows the distribution of the six risk choices paired according to the three trial types with dark shade representing choices with winning odds $<50 \%$ and light shade choices with winning odds $>50 \%$. Data presented for group means with $95 \%$ confidence interval error bars.

tion and image acquisition protocol, see Supplementary Information. Processing and statistical analysis of the functional images was performed in SPM8 (www.fil.ion.ucl. ac.uk/spm/software/spm8). The images were realigned to the first image in the time series, normalized to the MNI template (Montreal Neurological Institute), and smoothed using a symmetric 8-mm Gaussian kernel. The first level statistical analysis used event-related general linear models with 12 regressors of interest: 6 regressors for the choice phase ( 1 for each risk level, from the lowest odds $1 / 7$, to the highest odds 6/7) and 6 regressors for the outcome phase (3 for negative and 3 for positive events). Owing to different inter-individual bias in risk preference, a sufficient number of measurements for all types of outcome events was achieved by grouping the outcome events two-by-two. Thus, outcome events preceded by choices with odds of $1 / 7$ and 2/7 were modeled together as 'high-risk' events, outcome events preceded by choices with odds of $3 / 7$ and $4 / 7$ as 'mediumrisk' events, and outcome events preceded by choices with odds of 5/7 and 6/7 as 'low-risk' events. In addition to the six choice phase regressors and six outcome regressors, the model also included 24 nuisance regressors to correct for physiological noise related to head movement. Thus, three first-level contrasts of interest were computed based on the risk magnitude of the choice: (i) a risk choice contrast by assigning the six risk levels weights corresponding with a linear increase with the risk size, (ii) a contrast modeling the BOLD response to high positive outcomes (high-risk wins > low-risk wins), and (iii) contrast modeling the BOLD response for high negative outcome (low-risk loss $>$ high-risk loss). Because the loss amount was invariant across trials and matched the bet size, we differentiated negative outcomes by the value of the missed reward, which was high for low-risk and low for high-risk choices. We hypothesized that losing following a low-risk choice would be perceived more aversive compared with losing following a high-risk choice (Macoveanu et al, 2013a).

The three first level contrasts were included in separate $2 \times 2$ second level analysis of variance (ANOVA) models with factors: 'intervention' (GnRHa and placebo) and 'time' (baseline and follow-up). The models further included a 'subject' factor and main effect of time and group regressors to account for general differences between the two intervention groups that were not specific to the experimental task. Effects of intervention on the BOLD response were evaluated using second level intervention-by-time interaction contrasts: $\left[\mathrm{GnRHa}_{\text {baseline }}-\mathrm{GnRHa}_{\text {follow-up }}\right]-$ [Placebo $_{\text {baseline }}-$ Placebo $\left._{\text {follow-up }}\right]$ and $\left[\mathrm{GnRHa}_{\text {follow-up }}-\mathrm{GnRHa}_{\text {baseline }}\right]-\left[\right.$ Placebo $_{\text {follow-up }}$ Placebo $\left._{\text {baseline }}\right]$. Upon significant findings (see below), post hoc two-sided $t$-tests were used to evaluate the significance of group differences. As the decline in ovarian sex-steroid levels and increase in depression scores following the GnRHa treatment was expected to vary across subjects, we investigated possible correlations between changes in these factors and BOLD changes. We therefore set up three multiple regression models using the baseline $>$ follow-up first-level BOLD contrasts $(\triangle B O L D)$ as dependent variables and the factors that showed significant changes following the intervention ( $\triangle \mathrm{HAMD}, \Delta$ Estradiol, and $\Delta$ Testosterone) 
Table I Demographic and Clinical Data

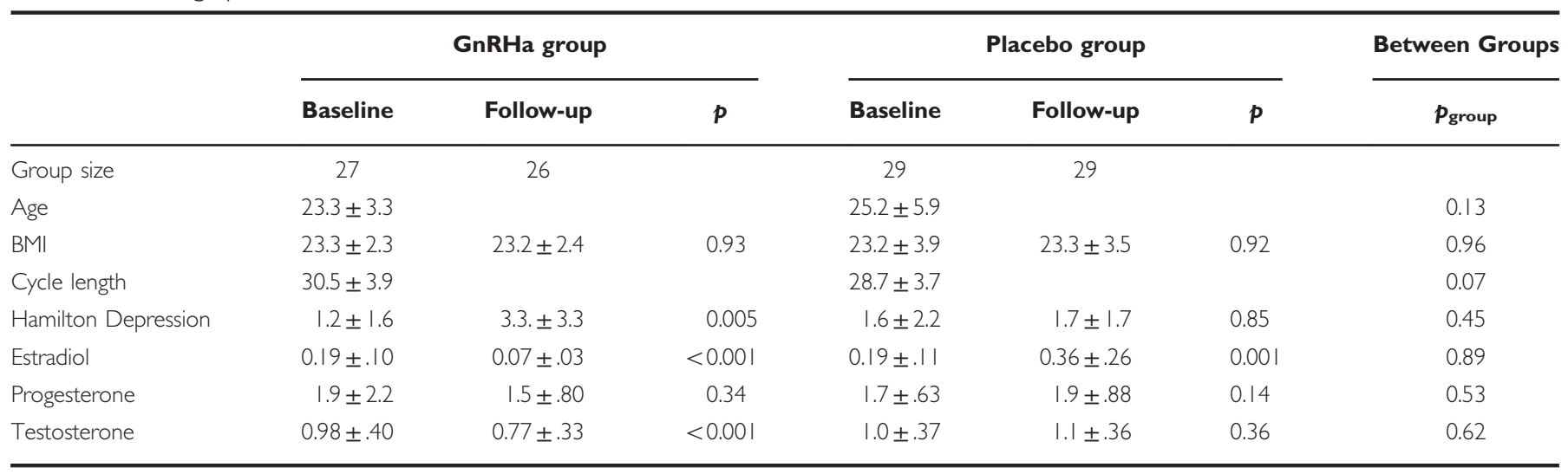

Mean values \pm SD. Hormone concentration are in nmol/l. p proup is the statistical value for the comparison between the GnRHa and placebo groups at baseline.

as covariates. We further explored $\triangle \mathrm{BOLD}-\Delta$ Progestorone correlations in a separate model.

Clusters were considered significant at $p<0.05$ after Family-Wise Error (FWE) correction for multiple nonindependent comparisons. The extent of each cluster was thresholded by applying an uncorrected voxel threshold of $p<0.005$. Amygdala was a priori defined as a region of interest (ROI) (see Introduction). Correction for multiple comparisons was therefore restricted to voxels within the ROI mask, which was constructed using the WFU PickAtlas toolbox (Tzourio-Mazoyer et al, 2002; Maldjian et al, 2003). Significant clusters are reported with Z-score and stereotactic MNI coordinates of the regional maxima $[\mathrm{x}, \mathrm{y}, \mathrm{z}]$.

\section{Evaluation of Choice Behavior during Gambling, Depressive Symptoms, and Estradiol Levels}

Risk-taking behavior during gambling was evaluated as the frequency of the different risk choices, which were entered into a repeated measures ANOVA model (PASW-SPSS20 statistics software, Chicago, IL, USA) with time of investigation (baseline and follow-up) and risk level (three levels, odds of $4 / 7,5 / 7$, and 6/7) as within-subject factors and type of intervention (GnRHa and placebo) as between-subject factor. To avoid including perfectly collinear data, we included only the frequencies of the $4 / 7,5 / 7$, and $6 / 7$ low-risk choices in the statistical model. The impact of the outcome of the immediately preceding trial on risk preference (assessed as the frequency of high-risk choices with odds $1 / 7,2 / 7$, and 3/7) was evaluated in an ANOVA with a time factor (baseline and follow-up), an intervention factor (GnRHa and placebo), a risk level factor (three levels: high, medium, and low risk, as defined above), and an outcome factor (negative and positive).

Absolute estradiol, progesterone, and testosterone levels and HAMD scores were evaluated in ANOVA models with 'time' as the within-subject factor and 'intervention' as the betweensubject factor. The general significance threshold was set at $p<0.05$ using the Greenhouse-Geisser correction for nonsphericity when appropriate. Conditional on significant F-values, pair-wise post hoc two-sided $t$-tests were performed to further explore significant main effects and interactions. We further explored possible correlations between the measurements showing a significant change from baseline to follow-up in the GnRHa-treated group by performing Pearson correlations.

\section{RESULTS}

\section{Effects of GnRHa on Hormone Levels and Depression Scores}

The GnRHa intervention decreased serum levels of estradiol and testosterone from baseline to follow-up as expected (Table 1). We further found increased Hamilton depression scores at follow-up, with changes observed across all symptom categories. GnRHa-associated decrease in absolute estradiol and testosterone levels were inter-correlated (Pearson correlation $=0.40, p=0.041, N=26$ ). The strict blinded design allowed for equal timing of baseline and follow-up with respect to phase (follicular), but not for cycle day, in the placebo group (Frokjaer et al, 2015). Accordingly, the placebo group was timed slightly later at follow-up and thus showed higher estradiol relative to baseline. There were no significant differences in the levels of progesterone, testosterone, or Hamilton scores in the placebo group at follow-up (Table 1). The individual baseline and follow-up values for the Hamilton scores, estradiol, and progesterone can be found in Frokjaer et al (2015).

\section{Gambling Behavior during fMRI}

Across all subjects, there was a trend towards more low-risk options compared with high-risk options $(\mathrm{F}(2,74)=3.3$, $p=0.053$, Figure 1c). The frequency of high-risk choices was influenced by both the magnitude of risk and the type of outcome of the immediately preceding trial (risk-by-outcome interaction: $F(2,52)=8.2, p=0.005)$. This interaction reflected a tendency towards increased risk-taking behavior following low-risk choices, which was most pronounced for low-risk choices resulting in a rewarding outcome. Compared with the placebo, the GnRHa intervention did not induce a consistent alteration in risk-taking behavior (groupby-time interaction: $\mathrm{F}(1,47)=0.006, p=0.9$ ).

\section{Neural Response to Risky Choices and Positive and Negative Outcomes at Baseline}

Replicating our previous work in gender-mixed cohorts (Macoveanu et al, 2013a,b), across all baseline measurements, the neural response during the choice phase increased linearly with the magnitude of risk of the chosen gamble in a 
widespread cortico-subcortical network, comprising the ventral striatum, insula, and anterior cingulate cortex (Table 2A). Positive outcome (large vs small rewards) were associated with increased activity in core regions of the reward system including the ventral striatum, amygdala, and insula (Table 2B). Negative outcomes (large $v s$ small missed rewards) were associated with increased activity in a bihemispherical network encompassing striatum, insula, anterior cingulate, and middle frontal cortex (Table 2C).

\section{The Effect of the GnRHa Intervention on Task-Related Activity}

The analysis of the brain response to high monetary rewards using the two-factorial ANOVA (between-group factor, intervention: placebo and GnRHa, within-subject factor, time: baseline and follow-up) revealed a significant intervention-by-time interaction in right amygdala (peak effect at $x, y, z=[28,-8,-16], Z=3.9, p_{F W E}=0.033$, corrected within amygdala $\mathrm{ROI}$ ) and a trend in the left amygdala (peak effect at $\mathrm{x}, \mathrm{y}, \mathrm{z}=[-26,-0,-16], \mathrm{Z}=2.9$, voxel $\left.p_{\text {uncorr }}<0.002\right)$. This interaction effect shows that the amygdala response to high monetary rewards was altered by the GnRHa intervention relative to placebo. Post hoc paired $t$-tests comparing baseline with follow-up in the GnRHa group revealed an attenuated response to reward magnitude in bilateral amygdala following $\mathrm{GnRHa}$ (peak effect right amygdala at $[30,-6,-18], Z=3.2, p_{\mathrm{FWE}}=0.062$ (statistical trend); left amygdala at $[-22,-8,-16], \mathrm{Z}=3.4, \mathrm{p}_{\mathrm{FWE}}=$ 0.023; corrected within amygdala ROI, Figure 2).

Relative to the placebo, the GnRHa intervention did not have a significant effect on the neural response to negative outcomes as signaled by high missed rewards or risky choices at whole brain level or within the amygdala ROI.

\section{Correlation Analyses between Individual Changes in Task-Related Activity and Changes in Hormonal Levels and Hamilton Scores in the GnRHa Group}

Positive outcomes. Overlapping with the brain network involved in processing positive outcomes (Table 1B), the exploratory whole-brain correlation analysis revealed bilateral insula regions showing a positive linear relationship between the magnitude of the GnRHa-induced change in testosterone levels (baseline $>$ to-follow-up) and BOLD changes (peak effect in right insula at $x, y, z=[38,-2,10]$, $\mathrm{Z}=4.2, \mathrm{p}_{\mathrm{FWE}}=0.017$, and left insula at $\mathrm{x}, \mathrm{y}, \mathrm{z}=[-36,-8,12]$, $\mathrm{Z}=3.8, \quad \mathrm{p}_{\mathrm{FWE}}=0.021$ ) (Figure 3 ). The left insula cluster extended into the putamen (secondary peak at $\mathrm{x}, \mathrm{y}$, $\mathrm{z}=[-30,-2,6], Z=3.5)$. Outside the task-related network, the GnRHa-induced testosterone changes correlated with BOLD-response changes in a cluster covering the right calcarine gyrus and extending into the middle temporal cortex (peak effect at $\mathrm{x}, \mathrm{y}, \mathrm{z}=[28,-54,12], \quad \mathrm{Z}=4.3$, $\left.\mathrm{p}_{\mathrm{FWE}}<0.001\right)$. No such correlation was observed within the amygdala ROI. It is noteworthy that the modulation of insula's response by testosterone is specific to the positive outcomes and was not present to negative outcomes or risky choices even at very liberal voxel threshold ( $p<0.01$ uncorrected). In contrast, the occipital region showed a trend correlation with testosterone changes during risky choices as well (peak effect at $\mathrm{x}, \mathrm{y}, \mathrm{z}=[16,-54,2], \mathrm{Z}=3.2, \mathrm{p}_{\mathrm{FWE}}>0.05$ n.s.). GnRHainduced changes in estradiol, progesterone, and Hamilton ratings did not correlate with change in reward-related activity at whole-brain level or within the amygdala ROI.

Negative outcomes. The GnRHa-induced changes in response to negative outcomes did not correlate with the changes in any of the measured hormones or depression ratings at whole brain level or within the amygdala ROI.

Risky choices. There was a positive correlation between the GnRHa-induced changes in the response to risky choices and changes in progesterone levels in the leg area of the primary motor cortex (peak effect in precentral gyrus at $\left.\mathrm{x}, \mathrm{y}, \mathrm{z}=[6,-32,76], \mathrm{Z}=4.5, \mathrm{p}_{\mathrm{FWE}}<0.001\right)$. The motor cortex region did not overlap with the task-related network (Table 1A), and at liberal voxel threshold $(p<0.01$ uncorrected), the changes in progesterone levels weakly correlated in this region with the BOLD-changes to positive outcomes as well (peak effect at $\mathrm{x}, \mathrm{y}, \mathrm{z}=[6,-34,78], \mathrm{Z}=2.5$, $\mathrm{p}_{\mathrm{FWE}}>0.05$ n.s.). The response to risky choices did not correlate with the GnRHa-induced changes in estradiol, testosterone, or Hamilton scores at whole brain level or within the amygdala ROI.

\section{DISCUSSION}

The GnRHa treatment in our cohort of young healthy women resulted in a significant increase in the Hamilton depression scores and a net decrease in the levels of estradiol and testosterone. Compared with the placebo group, the GnRHa intervention group showed a decreased amygdala response to the magnitude of monetary reward. The reduced amygdala responsivity was not associated with the magnitude of the net decline in estradiol or testosterone levels or increase in Hamilton scores. However, we found the individual GnRHa-induced decrease in testosterone levels to correlate positively with the decrease in insula response to positive outcomes bilaterally.

\section{Decreased Amygdala Engagement following the GnRHa Intervention}

There is a growing body of evidence on the influence of ovarian and exogenous sex steroids on cortical and subcortical regions involved in emotional and cognitive regulation (Toffoletto et al, 2014). Indeed, sex-steroid receptors are expressed in high densities in the amygdala and hippocampus, and are found to interact with other neurotransmitter systems, eg, serotonin and dopamine (Barth et al, 2015). Amygdala also expresses high levels of aromatase, the enzyme which regulates the last step of estradiol biosynthesis from testosterone (Biegon et al, 2010).

Amygdala has a key role in reward learning as well as processing positive and negative emotional reactions (Murray, 2007) being suggested to encode the stimulus reward value in concert with orbitofrontal cortex (Gottfried et al, 2003; Holland and Gallagher, 2004; O'Doherty, 2004). Natural variations in the hormonal milieu during the menstrual cycle have been shown to affect amygdala's reactivity to both positive and negative valence stimuli in several recent functional neuroimaging studies 
Table 2 fMRI Results across All Participants

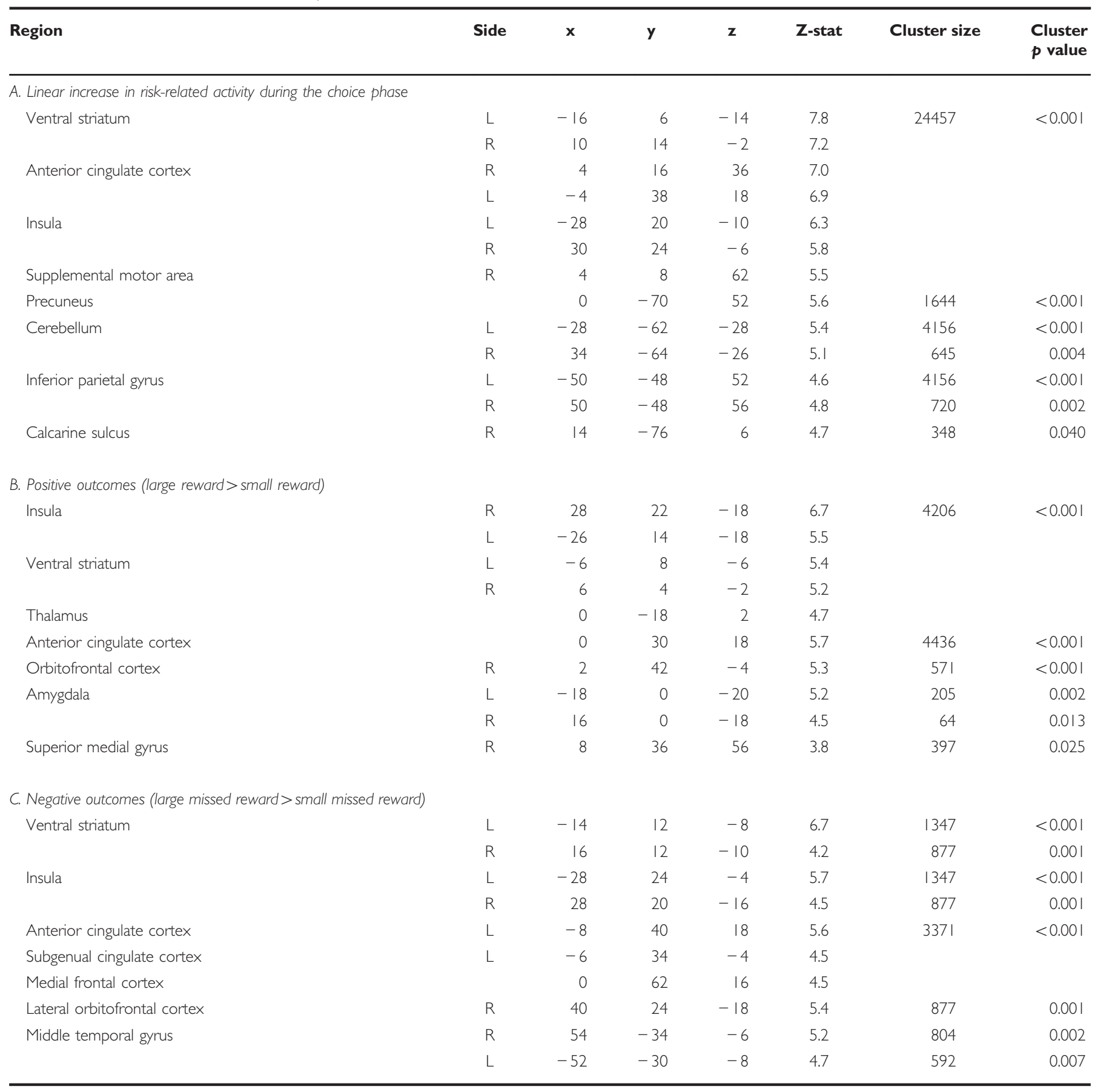

Data for regional peaks (cluster pFWE-corr $<0.05$; extent threshold $p \leq 0.001$ uncorrected). Coordinate $x, y, Z$ values in MNI standard stereotactic space, $Z$ statistics and cluster size in voxels.

(Goldstein et al, 2005; Ossewaarde et al, 2010; Gingnell et al, 2012; Bayer et al, 2014). Consistent with these findings, our data in healthy women show decreased amygdala response to the magnitude of the monetary gains following a rapid decline in ovarian sex-steroid levels. The observed reduction in amygdala's response was, however, not linear to the magnitude of the net decrease in sex steroids.

Testosterone administration in healthy middle-aged women was found to rapidly alter the functional coupling of the amygdala with the orbitofrontal cortex during emotional face processing, effect suggested to reflect a reduced regulatory control over the amygdala or that testosterone shifts amygdala output away from the orbitofrontal cortex towards the thalamus (van Wingen et al, 2010). In line with this view, acute changes in sex-steroid levels may alter the cortico-limbic top-down control of emotional responses reducing the reward value encoded by amygdala. 
Mood disorders are consistently associated with abnormalities in the processing of emotional stimuli with patients displaying an increased attentional bias towards negative cues and a decreased attentional bias towards positive cues (Leppänen, 2006). Recent neuroimaging studies have started to expose the neural underpinnings of the attentional bias observed in mood disorders, such as major depression. Depressed patients show an increased response to negative
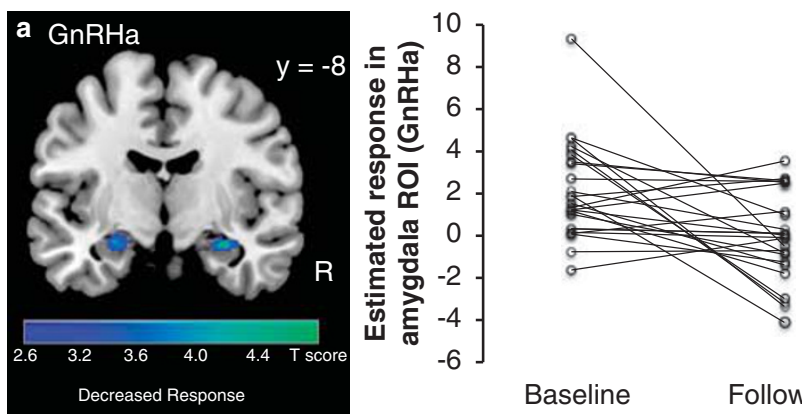

Baseline Follow-up
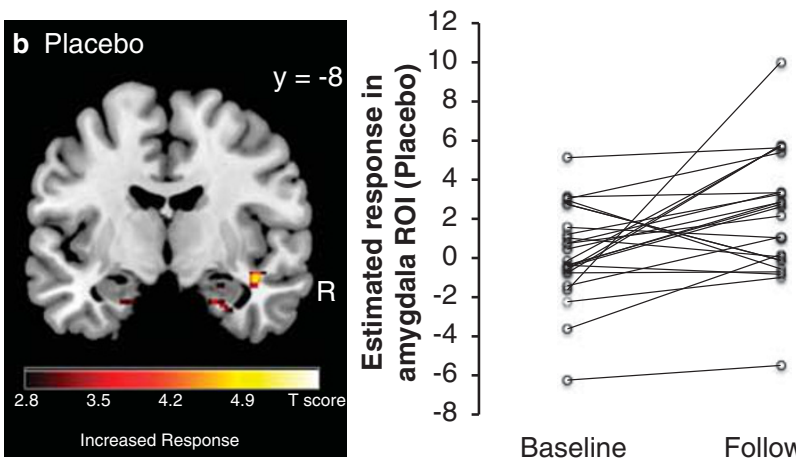

Baseline

Follow-up

Figure 2 Response in bilateral amygdala to positive monetary outcomes (high-risk > low-risk choices) following the GnRHa intervention and placebo. Left panel: color-coded statistical parametric maps thresholded at $p<0.005$ uncorrected. Right panel: Individual parameter estimates averaged within the amygdala ROI at baseline and following the GnRHa intervention.

cues in regions associated with reward and emotional processing, eg, ventral striatum and amygdala relative to healthy controls (Fu et al, 2004). Conversely, these regions show a decreased response to positive emotional cues (Victor et al, 2010; Stuhrmann et al, 2013). Interestingly, in a smaller group of major depression patients with varying degrees of anhedonia, Keedwell et al (2005) showed that anhedonia, and not depression severity per se, was coupled to the decreased response in amygdala and ventral striatum to positive valence stimuli. Our data support and extend these clinical findings suggesting that acute changes in sexhormone levels may contribute to the increased vulnerability for neuropsychiatric disorders in women by desensitizing amygdala's response to rewarding stimuli. This effect did, however, not lead to a significant change in risk-taking behavior, finding that may be explained by the stable neural response to risky choices from the baseline to the follow-up measurement.

\section{Proportional Change in Insula Response to Positive Outcomes and Change in Net Testosterone Levels in the GnRHa Group}

Neuroimaging studies have reliably shown insula's involvement in experimental tasks addressing risk-taking, monetary rewards, and punishment (Critchley et al, 2001; Kuhnen and Knutson, 2005; Clark et al, 2008; Preuschoff et al, 2008; Studer et al, 2012). An abnormal top-down regulation of emotional processing in the insular cortex has been proposed to have a key role in the pathophysiology of depression (Sliz and Hayley, 2012), which is associated with a blunted emotional response to normally pleasant or rewarding stimuli (Snaith, 1993). In line with this, in a meta-analysis study, Fitzgerald et al (2008) report consistent insula hypoactivity in depression (among other regions) in resting state paradigms and emotional activation studies.
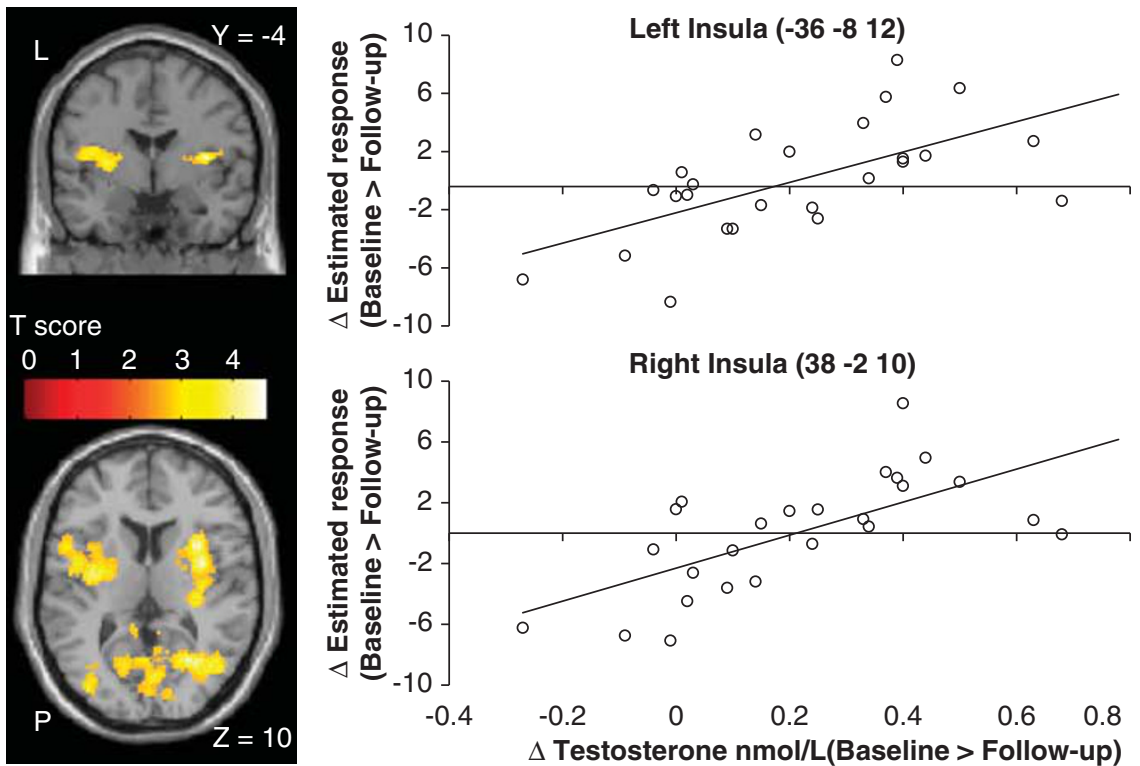

Right Insula (38 -2 10)

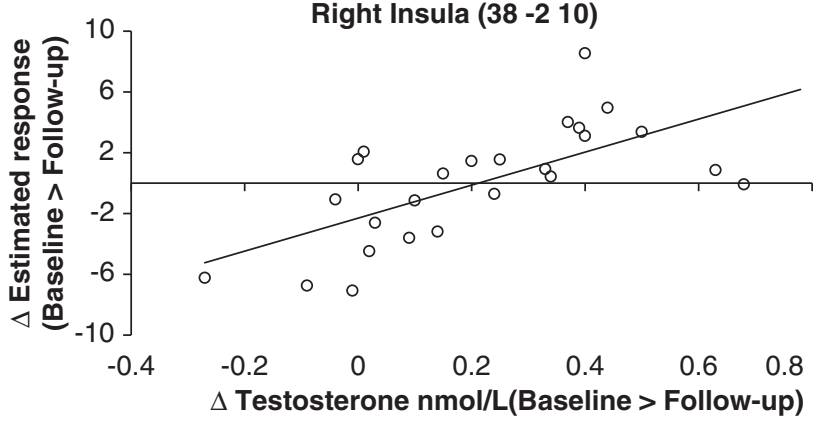

Figure 3 Color-coded statistical parametric maps of brain regions showing a positive linear relationship between the individual net testosterone changes (baseline $>$ follow-up) and changes in reward-related response in the GnRHa group. Extent threshold $p<0.005$ uncorrected. 
Although insula's involvement in risk-taking and reward is well established, the role testosterone has in modulating these brain processes is not well understood. Initial findings in adolescent boys and girls suggest a link between individual absolute testosterone level, orbitofrontal cortex development, and risk-taking behavior with a positive correlation between the magnitude of risk and endogenous testosterone levels (Peper et al, 2013). However, the impact of testosterone on the pathogenesis of psychiatric disorders is supported by several clinical studies (Höfer et al, 2013). Specifically, major depression in women has been associated with lower absolute testosterone levels (Oulis et al, 2014), and there is a wide range of clinical studies documenting significant positive effect of testosterone therapy on HAMD rating in depressed patients when compared with placebo (Zarrouf et al, 2009; Amanatkar et al, 2014).

Although the GnRHa intervention did not result in a significant overall reduction in insula's reward-related activity across the studied group, we found the magnitude of the individual GnRHa-induced changes in net testosterone levels to be predictive of changes in insula's response to rewards. To our knowledge, our study is the first to demonstrate a link between fluctuations in testosterone levels and reward-related insular activity in parallel with increased depression symptoms. These findings may advance the understanding of the role sex-steroid biology has in depression by providing a plausible biological mechanism for the altered reward-related insula response in depression.

Although testosterone modulated the reward response in both insula and occipital cortex, only the insular response was overlapping with the reward network and was specific to positive outcomes. Similarly, progesterone was found not only to modulate primary motor cortex response to risky choices but also weakly to positive outcomes. The findings in occipital and motor cortex are likely to represent nonspecific effects on sensory and motor processing mediated by the changes in sex-steroid levels.

\section{Limitations}

Our investigation only measures the total (protein and nonprotein bound) fractions of the sex-steroid hormones and not directly their biologically active form. However, the total hormone values are considered to index well the biologically active fraction particularly in the current population of healthy women with normal BMI, no diabetes, and normal thyroid-stimulating hormone. Further, our test battery did not include a direct measure of anhedonia. We are therefore not able to directly relate the observed blunted rewardrelated response to increased anhedonic behavior.

\section{CONCLUSIONS}

Taken together, our data provide strong evidence for ovarian sex-steroid hormone involvement in the functional modulation of the reward system. Following an acute decline in the level of sex-steroid hormones, we found a blunted response in amygdala to monetary rewards, a key correlate of depression. We further demonstrate a positive correlation between the decrease in testosterone levels and decrease in insula response to monetary rewards. These findings may represent key mechanism by which sex-steroid fluctuations provoke mood disorders in susceptible women. This insight may translate to preventive strategies, eg, in women at high risk during perimenopause or the immediate postpartum period.

\section{FUNDING AND DISCLOSURE}

As a potential conflict of interest HRS declares that he has received honoraria as Handling and Senior Editor for NeuroImage, as speaker for Biogen Idec Denmark A/S, Genzyme Denmark and MerckSerono Denmark, and scientific Advisor from Lundbeck A/S, Valby, Denmark, and has received financial travel support by Magventure, Skovlunde, Denmark. The other authors declare no conflict of interests.

\section{ACKNOWLEDGMENTS}

The study was funded by The Danish Council for Independent Research, the Lundbeck Foundation, and The Capital Region of Denmark, foundation for Health Research.

\section{REFERENCES}

Amanatkar HR, Chibnall JT, Seo B-W, Manepalli JN, Grossberg GT (2014). Impact of exogenous testosterone on mood: a systematic review and meta-analysis of randomized placebo-controlled trials. Ann Clin Psychiatry 26: 19-32.

Barth C, Villringer A, Sacher J (2015). Sex hormones affect neurotransmitters and shape the adult female brain during hormonal transition periods. Front Neurosci 9: 37.

Bayer J, Bandurski P, Sommer T (2013). Differential modulation of activity related to the anticipation of monetary gains and losses across the menstrual cycle. Eur J Neurosci 38: 3519-3526.

Bayer J, Schultz H, Gamer M, Sommer T (2014). Menstrual-cycle dependent fluctuations in ovarian hormones affect emotional memory. Neurobiol Learn Mem 110: 55-63.

Biegon A, Kim SW, Alexoff DL, Jayne M, Carter P, Hubbard B et al (2010). Unique distribution of aromatase in the human brain: in vivo studies with PET and [N-methyl-11C]vorozole. Synapse 64: 801-807.

Brummelte S, Galea LA (2010). Depression during pregnancy and postpartum: contribution of stress and ovarian hormones. Prog Neuropsychopharmacol Biol Psychiatry 34: 766-776.

Clark DA, Beck AT, Beck JS (1994). Symptom differences in major depression, dysthymia, panic disorder, and generalized anxiety disorder. Am J Psychiatry 151: 205-209.

Clark L, Bechara A, Damasio H, Aitken MR, Sahakian BJ, Robbins TW (2008). Differential effects of insular and ventromedial prefrontal cortex lesions on risky decision-making. Brain 131: 1311-1322.

Critchley HD, Mathias CJ, Dolan RJ (2001). Neural activity in the human brain relating to uncertainty and arousal during anticipation. Neuron 29: 537-545.

Dreher J-C, Schmidt PJ, Kohn P, Furman D, Rubinow D, Berman KF (2007). Menstrual cycle phase modulates reward-related neural function in women. Proc Natl Acad Sci USA 104: 2465-2470.

Fitzgerald PB, Laird AR, Maller J, Daskalakis ZJ (2008). A metaanalytic study of changes in brain activation in depression. Hum Brain Mapp 29: 683-695. 
Freeman EW, Sammel MD, Lin H, Nelson DB (2006). Associations of hormones and menopausal status with depressed mood in women with no history of depression. Arch Gen Psychiatry 73: 375-382.

Freeman EW, Sammel MD, Liu L, Gracia CR, Nelson DB, Hollander L (2004). Hormones and menopausal status as predictors of depression in women in transition to menopause. Arch Gen Psychiatry 61: 62-70.

Frokjaer VG, Pinborg A, Holst KK, Overgaard A, Henningsson S, Heede $M$ et al (2015). Role of serotonin transporter changes in depressive responses to sex-steroid hormone manipulation: a positron emission tomography study. Biol Psychiatry (e-pub ahead of print 27 April 2015).

Fu CHY, Williams SCR, Cleare AJ, Brammer MJ, Walsh ND, Kim J et al (2004). Attenuation of the neural response to sad faces in major depression by antidepressant treatment: a prospective, event-related functional magnetic resonance imaging study. Arch Gen Psychiatry 61: 877-889.

Gingnell M, Morell A, Bannbers E, Wikström J, Sundström Poromaa I (2012). Menstrual cycle effects on amygdala reactivity to emotional stimulation in premenstrual dysphoric disorder. Horm Behav 62: 400-406.

Goldstein JM, Jerram M, Poldrack R, Ahern T, Kennedy DN, Seidman LJ et al (2005). Hormonal cycle modulates arousal circuitry in women using functional magnetic resonance imaging. J Neurosci 25: 9309-9316.

Gottfried JA, O’Doherty J, Dolan RJ (2003). Encoding predictive reward value in human amygdala and orbitofrontal cortex. Science 301: 1104-1107.

Hamilton M (1980). Rating depressive patients. J Clin Psychiatry 41: 21-24.

Höfer P, Lanzenberger R, Kasper S (2013). Testosterone in the brain: neuroimaging findings and the potential role for neuropsychopharmacology. Eur Neuropsychopharmacol 23: 79-88.

Holland PC, Gallagher M (2004). Amygdala-frontal interactions and reward expectancy. Curr Opin Neurobiol 14: 148-155.

Keedwell PA, Andrew C, Williams SC, Brammer MJ, Phillips ML (2005). The neural correlates of anhedonia in major depressive disorder. Biol Psychiatry 58: 843-853.

Kuhnen CM, Knutson B (2005). The neural basis of financial risk taking. Neuron 47: 763-770.

Leppänen JM (2006). Emotional information processing in mood disorders: a review of behavioral and neuroimaging findings. Curr Opin Psychiatry 19: 34-39.

Macoveanu J, Rowe JB, Hornboll B, Elliott R, Paulson OB, Knudsen GM et al (2013a). Playing it safe but losing anyway-Serotonergic signaling of negative outcomes in dorsomedial prefrontal cortex in the context of risk-aversion. Eur Neuropsychopharmacol 23: 919-930.

Macoveanu J, Rowe JB, Hornboll B, Elliott R, Paulson OB, Knudsen GM et al (2013b). Serotonin $2 \mathrm{~A}$ receptors contribute to the regulation of risk-averse decisions. Neuroimage 83: $35-44$

Maldjian JA, Laurienti PJ, Kraft RA, Burdette JH (2003). An automated method for neuroanatomic and cytoarchitectonic atlas-based interrogation of fMRI data sets. Neuroimage 19: 1233-1239.
Munk-Olsen T, Laursen TM, Pedersen CB, Mors O, Mortensen PB (2006). New parents and mental disorders: a population-based register study. JAMA 296: 2582-2589.

Murray EA (2007). The amygdala, reward and emotion. Trends Cogn Sci 11: 489-497.

O’Doherty JP (2004). Reward representations and reward-related learning in the human brain: insights from neuroimaging. Curr Opin Neurobiol 14: 769-776.

Ossewaarde L, Hermans EJ, van Wingen GA, Kooijman SC, Johansson I-M, Bäckström T et al (2010). Neural mechanisms underlying changes in stress-sensitivity across the menstrual cycle. Psychoneuroendocrinology 35: 47-55.

Oulis P, Masdrakis VG, Markianos M (2014). Testosterone and dehydroepiandrosterone sulfate in female anxious and nonanxious major depression. Int J Psychiatry Clin Pr 18: 21-24.

Peper JS, Koolschijn PC, Crone EA (2013). Development of risk taking: Contributions from adolescent testosterone and the orbito-frontal cortex. J Cogn Neurosci 25: 2141-2150.

Preuschoff K, Quartz SR, Bossaerts P (2008). Human insula activation reflects risk prediction errors as well as risk. $J$ Neurosci 28: $2745-2752$.

Sliz D, Hayley S (2012). Major depressive disorder and alterations in insular cortical activity: a review of current functional magnetic imaging research. Front Hum Neurosci 6: 323.

Snaith P (1993). Anhedonia: a neglected symptom of psychopathology. Psychol Med 23: 957-966.

Studer B, Apergis-Schoute AM, Robbins TW, Clark L (2012). What are the odds? The neural correlates of active choice during gambling. Front Neurosci 6: 46.

Stuhrmann A, Dohm K, Kugel H, Zwanzger P, Redlich R, Grotegerd D et al (2013). Mood-congruent amygdala responses to subliminally presented facial expressions in major depression: associations with anhedonia. J Psychiatry Neurosci 38: 249-258.

Toffoletto S, Lanzenberger R, Gingnell M, Sundström-Poromaa I, Comasco E (2014). Emotional and cognitive functional imaging of estrogen and progesterone effects in the female human brain: A systematic review. Psychoneuroendocrinology 50C: 28-52.

Tzourio-Mazoyer N, Landeau B, Papathanassiou D, Crivello F, Etard O, Delcroix N et al (2002). Automated anatomical labeling of activations in SPM using a macroscopic anatomical parcellation of the MNI MRI single-subject brain. Neuroimage 15: 273-289.

Victor TA, Furey ML, Fromm SJ, Ohman A, Drevets WC (2010). Relationship between amygdala responses to masked faces and mood state and treatment in major depressive disorder. Arch Gen Psychiatry 67: 1128-1138.

Vrieze E, Pizzagalli DA, Demyttenaere K, Hompes T, Sienaert P, de Boer P et al (2013). Reduced reward learning predicts outcome in major depressive disorder. Biol Psychiatry 73: 639-645.

van Wingen G, Mattern C, Verkes RJ, Buitelaar J, Fernández G (2010). Testosterone reduces amygdala-orbitofrontal cortex coupling. Psychoneuroendocrinology 35: 105-113.

Zarrouf FA, Artz S, Griffith J, Sirbu C, Kommor M (2009). Testosterone and depression: systematic review and metaanalysis. J Psychiatr Pr 15: 289-305.

Supplementary Information accompanies the paper on the Neuropsychopharmacology website (http://www.nature.com/npp) 